\title{
Investigation of Flow Through Centrifugal Pump Impellers Using Computational Fluid Dynamics
}

\author{
Weidong Zhou, Zhimei Zhao, T. S. Lee, and S. H. Winoto \\ Fluid Mechanics Laboratory, Mechanical Engineering Department, National University of Singapore, \\ Singapore
}

With the aid of computational fluid dynamics, the complex internal flows in water pump impellers can be well predicted, thus facilitating the design of pumps. This article describes the three-dimensional simulation of internal flow in three different types of centrifugal pumps (one pump has four straight blades and the other two have six twisted blades). A commercial three-dimensional Navier-Stokes code called CFX, with a standard $k-\epsilon$ two-equation turbulence model was used to simulate the problem under examination. In the calculation, the finite-volume method and an unstructured grid system were used for the solution procedure of the discretized governing equations for this problem.

Comparison of computational results for various types of pumps showed good agreement for the twisted-blade pumps. However, for the straight-blade pump, the computational results were somewhat different from widely published experimental results. It was found that the predicted results relating to twisted-blade pumps were better than those relating to the straight-blade pump, which suggests that the efficiency of a twisted-blade pump will be greater than that of a straight-blade pump. The calculation also predicts reasonable results in both the flow pattern and the pressure distribution.

Keywords centrifugal pump, computational fluid dynamics, NavierStokes code, off-design condition, pump performance, unstructured mesh

Computational fluid dynamics (CFD) analysis is being increasingly applied in the design of centrifugal pumps. With the

Received 24 December 2001; accepted 11 January 2002.

Address correspondence to Zhou Weidong, Fluid Mechanics Laboratory, Mechanical Engineering Department, National University of Singapore, Singapore 119260, Singapore. E-mail: zhouwd@ hotmail. com aid of the CFD approach, the complex internal flows in water pump impellers, which are not fully understood yet, can be well predicted, to speed up the pump design procedure. Thus, CFD is an important tool for pump designers.

Many CFD studies concerning the complex flow in all types of centrifugal pumps have been reported. Oh and Ro (2000) used a compressible time marching method, a traditional SIMPLE method, and a commercial program of CFX-TASCflow to simulate flow pattern through a water pump and compared the differences among these methods in predicting the pump's performance.

Goto (1992) presented a comparison between the measured and computed exit-flow fields of a mixed flow impeller with various tip clearances, including the shrouded and unshrouded impellers, and confirmed the applicability of the incompressible version of the three-dimensional Navier-Stokes code developed by Dawes (1986) for a mixed-flow centrifugal pump.

Zhou and $\mathrm{Ng}$ (1998) and $\mathrm{Ng}$ and colleagues (1998) also developed a three-dimensional time-marching, incompressible Navier-Stokes solver using the pseudocompressibility technique to study the flow field through a mixed-flow water-pump impeller. The applicability of the original code was validated by comparing it with many published experimental and computational results.

Recently, Kaupert and colleagues (1996), Potts and Newton (1998), and Sun and Tsukamoto (2001) studied pump off-design performance using the commercial software CFX-TASCflow, FLUENT, and STARCD, respectively. Although these researchers predicted reverse flow in the impeller shroud region at small flow rates numerically, some contradictions still existed. For example, Kaupert's experiments showed the simultaneous appearance of shroud-side reverse flow at the impeller inlet and outlet, but his CFD results failed to predict the numerical outletreverse flow. Sun and Tsukamoto (2001) validated the predicted results of the head-flow curves, diffuser inlet pressure distribution, and impeller radial forces by revealing the experimental data over the entire flow range, and they predicted back flow 
at small flow rates, but they did not show an exact back-flow pattern along the impeller outlet.

From such literature, it was found that most previous research, especially research based on numerical approaches, had focused on the design or near-design state of pumps. Few efforts were made to study the off-design performance of pumps. Centrifugal pumps are widely used in many applications, so the pump system may be required to operate over a wide flow range in some special applications. Thus, knowledge about off-design pump performance is a necessity. On the other hand, it was found that few researchers had compared flow and pressure fields among different types of pumps. Therefore, there is still a lot of work to be done in these fields.

In this article, a commercial CFD code, called CFX, was used to study three-dimensional turbulent flow through water-pump impellers during design and off-design conditions. CFX is a software package that can predict laminar flow, turbulent flow, and heat transfer. It has been widely used in the field of turbomachinery, and the simulation results have been proven by many researchers to be reliable (Anderson et al., 2000; Miyazoe et al., 1999; Tatebayashi et al., 2000). CFX overcomes the meshing difficulties that arise in complex geometry by using a powerful CAD-based preprocessor, CFX-Build, which generates a surface mesh of triangles. This surface mesh is then converted into a volume mesh of tetrahedral elements by the flow solver.

Three different types of centrifugal pumps are considered in this simulation. One pump had four straight blades and the other two had six twisted blades. The predicted results for the headflow curves in these cases are presented over the entire flow range. The calculated results for velocity and pressure are also shown.

\section{MATHEMATICAL MODELS}

\section{Basic Equations}

For three-dimensional incompressible, unsteady flow, the continuity and momentum equations can be written in the rotating coordinate system as follows:

and

$$
\frac{\partial \rho}{\partial t}+\nabla \cdot(\rho U)=0
$$

$$
\begin{aligned}
& \frac{\partial \rho U}{\partial t}+\nabla \cdot(\rho U \otimes U) \\
& \quad=\nabla \cdot\left(-P \delta+\mu_{e f f}\left(\nabla U+(\nabla U)^{T}\right)\right)+S_{M} .
\end{aligned}
$$

Where vector notation has been used, $\otimes$ is a vector crossproduct; $U$ is the velocity; $P$ is the pressure; $\rho$ is the density; $\delta$ is the identity matrix; and $S_{M}$ is the source term.

For flows in a rotating frame of reference that are rotating at the constant rotation speed $\vec{\Omega}$, the effects of the Coriolis are modeled in the code. In this case,

$$
S_{M}=-\rho[2 \vec{\Omega} \otimes U+\vec{\Omega} \otimes(\vec{\Omega} \otimes \vec{r})]
$$

where $\vec{r}$ is the location vector.

\section{$\boldsymbol{k}-\varepsilon$ Turbulence Model}

In Equation (2), $\mu_{\text {eff }}$ is the effective viscosity coefficient, which equals the molecular viscosity coefficient, $\mu$, plus the turbulent eddy viscosity coefficient, $\mu_{t}$ :

$$
\mu_{\text {eff }}=\mu+\mu_{t}
$$

The turbulent viscosity, $\mu_{t}$, is modeled as the product of a turbulent velocity scale, $V_{t}$, and a turbulent length scale, $l_{t}$, as proposed by Kolmogorov (1941). Introducing a proportionality constant gives

$$
\mu_{t}=\rho c_{\mu} l_{t} V_{t}
$$

Both equation models take the velocity scale, $V_{t}$, to be the square root of the turbulent kinetic energy:

$$
V_{t}=\sqrt{k}
$$

The turbulent kinetic energy, $k$, is determined from the solution of a semiempirical transport equation.

In the standard $k-\varepsilon$ two-equation model it is assumed that the length scale is a dissipation length scale, and when the turbulent dissipation scales are isotropic, Kolmogorov determined that

$$
\varepsilon=\frac{k^{3 / 2}}{l_{t}}
$$

where $\varepsilon$ is the turbulent dissipation rate.

Therefore, the turbulence viscosity, $\mu_{t}$, can be derived from Equations (5), (6), and (7) to link to the turbulence kinetic energy and dissipation via the relation

$$
\mu_{t}=C_{\mu} \rho \frac{k^{2}}{\varepsilon}
$$

where $C_{\mu}$ is a constant. Its value is 0.09 .

The values of $k, \varepsilon$ come directly from the differential transport equations for the turbulence kinetic energy and turbulence dissipation rate:

$$
\frac{\partial \rho k}{\partial t}+\nabla \cdot(\rho U k)-\nabla \cdot\left(\Gamma_{k} \nabla k\right)=p_{k}-\rho \varepsilon
$$

and

$$
\frac{\partial \rho \varepsilon}{\partial t}+\nabla \cdot(\rho U \varepsilon)-\nabla \cdot\left(\Gamma_{\varepsilon} \nabla \varepsilon\right)=\frac{\varepsilon}{k}\left(C_{\varepsilon 1} p_{k}-C_{\varepsilon 2} \rho \varepsilon\right)
$$

where the diffusion coefficients are given by

$$
\Gamma_{k}=\mu+\frac{\mu_{t}}{\sigma_{k}}
$$

and

$$
\Gamma_{\epsilon}=\mu+\frac{\mu_{t}}{\sigma_{\varepsilon}}
$$

and $C_{\varepsilon 1}=1.44 ; C_{\varepsilon 2}=1.92 ; \sigma_{k}=1.0 ;$ and $\sigma_{\varepsilon}=1.3$ are constants. 
The $p_{k}$ in Equations (9) and (10) is the turbulent kinetic energy production term, which for incompressible flow is

$$
p_{k}=\mu_{t} \nabla U \cdot\left(\nabla U+\nabla U^{T}\right)-\frac{2}{3} \nabla \cdot U\left(\mu_{t} \nabla \cdot U+\rho k\right)
$$

Equations (1), (2), (9), and (10) form a closed set of nonlinear partial differential equations governing the fluid motion.

\section{Log-Law Wall Functions}

There are large gradients in the dependent variables near the wall. It is costly to fully resolve the solution in this near-wall region as the required number of nodes would be quite large. Thus a common approach known as "wall functions" is applied to model this region.

In the wall-function approach (Launder and Spalding 1974), the near wall tangential velocity is related to the wall shear stress by means of a logarithmic relation, which can be written as follows:

$$
u^{+}=\frac{1}{\kappa} \ln \left(y^{+}\right)+C
$$

where

$$
\begin{aligned}
u^{+} & =\frac{u_{t}}{u_{\tau}}, \\
y^{+} & =\frac{\rho \Delta y u_{\tau}}{\mu}, \\
u_{\tau} & =\left(\frac{\tau_{w}}{\rho}\right)^{1 / 2}
\end{aligned}
$$

$\tau_{w}$ is the wall shear stress,

$u_{t}$ is the known velocity tangent to the wall at a distance of $\Delta y$

from the wall,

$\kappa$ is the Von Karman constant for smooth walls, and

$\kappa$ and $C$ are constants, depending on wall roughness.

However, this form of the wall-function equations has the problem that it becomes singular at separation points where the near-wall velocity, $u_{t}$, approaches zero. In the logarithmic region, the alternative velocity scale, $u^{*}$, can be used instead of $u^{+}$:

$$
u^{*}=c_{\mu}^{1 / 4} \sqrt{k}
$$

This scale has the useful property of not going to zero if $u_{t}$ goes to zero (and in turbulent flow, $k$ is never completely zero). Based on this definition, the following explicit equation for the wall shear stress is obtained:

$$
\tau_{w}=\tau_{v i s c} \frac{y^{*}}{u^{+}}
$$

where

$$
\tau_{\mathrm{visc}}=\mu u_{t} / \Delta y ; \quad y^{*}=\rho u^{*} \Delta y / \mu ; \text { and } u^{+}=\frac{1}{\kappa} \ln \left(y^{*}\right)+C
$$

The recommended practice is to locate near-wall nodes such that $y^{*}$ is in the range of 20 to 50 for smooth walls. In the near- wall region, an estimate of the dissipation consistent with the log-law can be presented as

$$
\varepsilon=\frac{c_{\mu}^{3 / 4} k^{3 / 2}}{\kappa \Delta y}
$$

The dissipation at the first interior node is set equal to this value. The boundary nodal value for $k$ is estimated via an extrapolation boundary condition.

The near-wall production of turbulent kinetic energy is derived to be

$$
p_{k}=\frac{\tau_{v i s c}^{2}}{\mu} p_{k}^{*}
$$

where

$$
p_{k}^{*}=\left(\frac{y^{*}}{u^{+}}\right)^{2} \frac{d u^{+}}{d y^{*}}
$$

\section{COMPUTATIONAL GRID AND BOUNDARY CONDITIONS}

\section{Computational Grid}

Currently, the computations are performed on a centrifugal pump with four straight blades (M1), a centrifugal pump with six twisted blades (M2), and a centrifugal pump with six twisted blades of different sizes (M3). For pump M1, the design operating point is $\mathrm{n}=2900 \mathrm{rpm}, \mathrm{Q}=20 \mathrm{~m}^{3} / \mathrm{hr} ; \mathrm{n}=1450 \mathrm{rpm}$, $\mathrm{Q}=10 \mathrm{~m}^{3} / \mathrm{hr}$. For pump M2, the design operating point is $\mathrm{n}=$ $2900 \mathrm{rpm}, \mathrm{Q}=360 \mathrm{~m}^{3} / \mathrm{hr} ; \mathrm{n}=1450 \mathrm{rpm}, \mathrm{Q}=180 \mathrm{~m}^{3} / \mathrm{hr}$. For pump M3, the design operating point is $\mathrm{n}=2900 \mathrm{rpm}, \mathrm{Q}=$ $80 \mathrm{~m}^{3} / \mathrm{hr} ; \mathrm{n}=1450 \mathrm{rpm}, \mathrm{Q}=40 \mathrm{~m}^{3} / \mathrm{hr}$.

Figure 1 shows the three-dimensional pump geometry for each pump. As a preliminary study, only three-dimensional water flow through pump impellers was dealt with.

The unstructured triangular meshes were generated by CFX preprocessor-CFX-Build, as shown in Figure 2. The detailed grid system for each pump is presented in Table 1. Relatively fine grids were used near inlet, outlet, and wall surface, whereas the grids in other regions were coarse. The total computational time for the grid use of M1 and M2 was approximately $3 \mathrm{hr}$ of CPU time on the Compaq GS320 alphaserver.

A relatively coarse mesh was applied in the case of M3 because when we performed a mesh-independent check to the M2 case, it was found that a coarse mesh (around 6000-10,000 total elements) was enough to predict the pump H-Q curve and the flow patterns through the pump impellers. Therefore, this kind of coarse mesh was adopted to save CPU time. The total computational time for grid use was only about $30 \mathrm{~min}$ of CPU time. Table 2 presents the results of the mesh-independent check. Pump M2 was selected for this study. The operating point was $\mathrm{n}=1450 \mathrm{rpm}, \mathrm{Q}=180 \mathrm{~m}^{3} / \mathrm{hr}$. 
TABLE 1

Grid System for Each Pump Case

\begin{tabular}{lrrrrc}
\hline $\begin{array}{l}\text { Pump } \\
\text { case }\end{array}$ & $\begin{array}{c}\text { Total } \\
\text { elements }\end{array}$ & $\begin{array}{c}\text { Number } \\
\text { of nodes }\end{array}$ & $\begin{array}{c}\text { Number of } \\
\text { tetrahedra }\end{array}$ & $\begin{array}{c}\text { Number } \\
\text { of prisms }\end{array}$ & $\begin{array}{c}\text { Number of } \\
\text { pyramids }\end{array}$ \\
\hline M1 & 36707 & 11817 & 24018 & 12211 & 478 \\
M2 & 29188 & 9909 & 17325 & 11373 & 490 \\
M3 & 6065 & 2180 & 3535 & 2159 & 371 \\
\hline
\end{tabular}

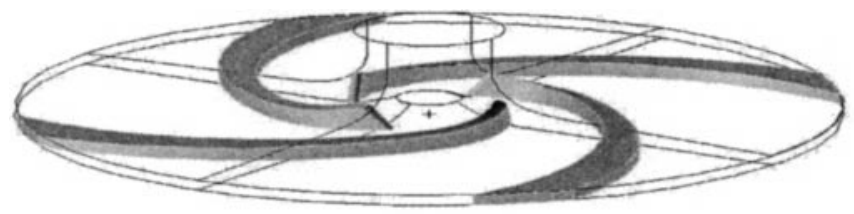

(a)

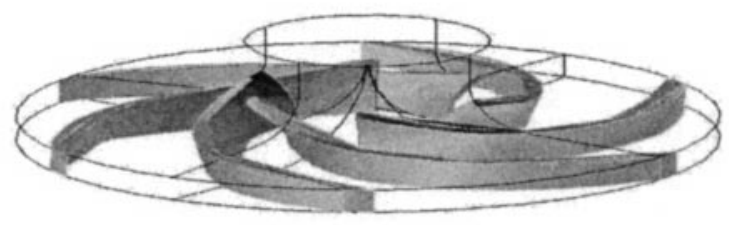

(b)

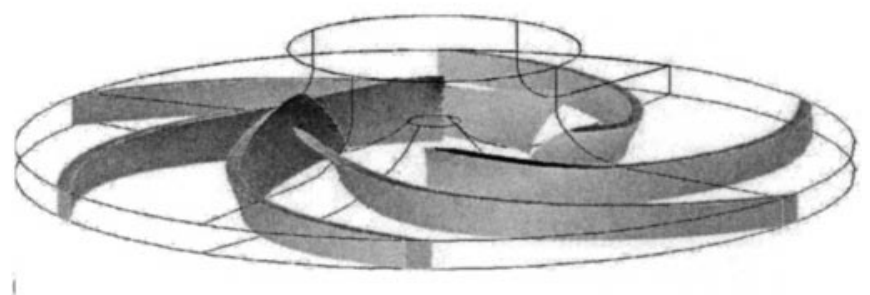

(c)

FIGURE 1

Three-dimensional geometry for pumps. (a) Pump M1. (b) Pump M2. (c) Pump M3.
TABLE 2

Results of Mesh Independent Check

\begin{tabular}{lcc}
\hline Case number & $\begin{array}{c}\text { Total mesh } \\
\text { number }\end{array}$ & $\begin{array}{c}\text { Calculated pump head } \\
(\mathrm{m})\end{array}$ \\
\hline Case 1 & 29190 & 31.781 \\
Case 2 & 9420 & 32.026 \\
Case 3 & 6462 & 32.495 \\
\hline
\end{tabular}

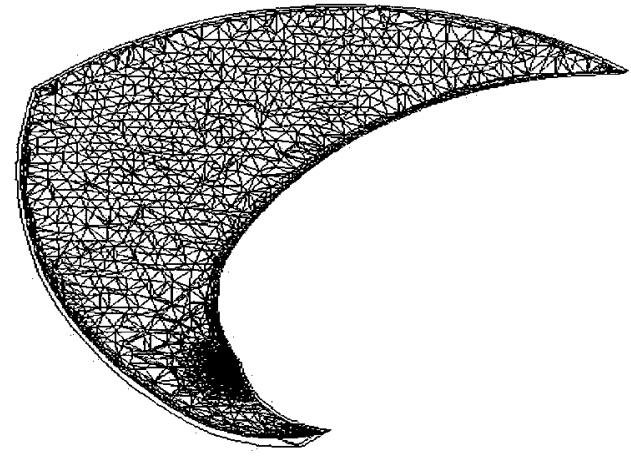

(a)

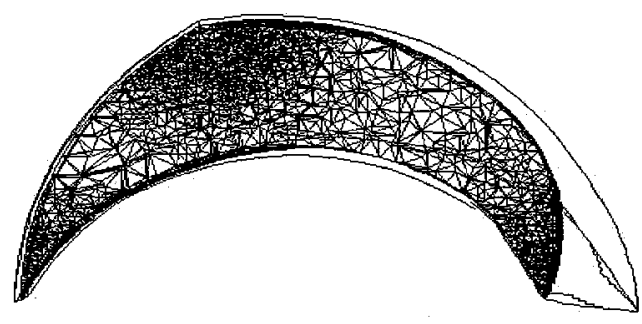

(b)

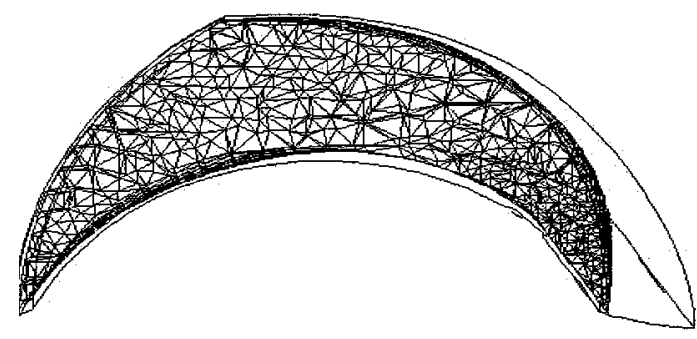

(c)

FIGURE 2

Computational grids for pumps generated by CFX-BUILD. (a) Pump M1. (b) Pump M2. (c) Pump M3. 


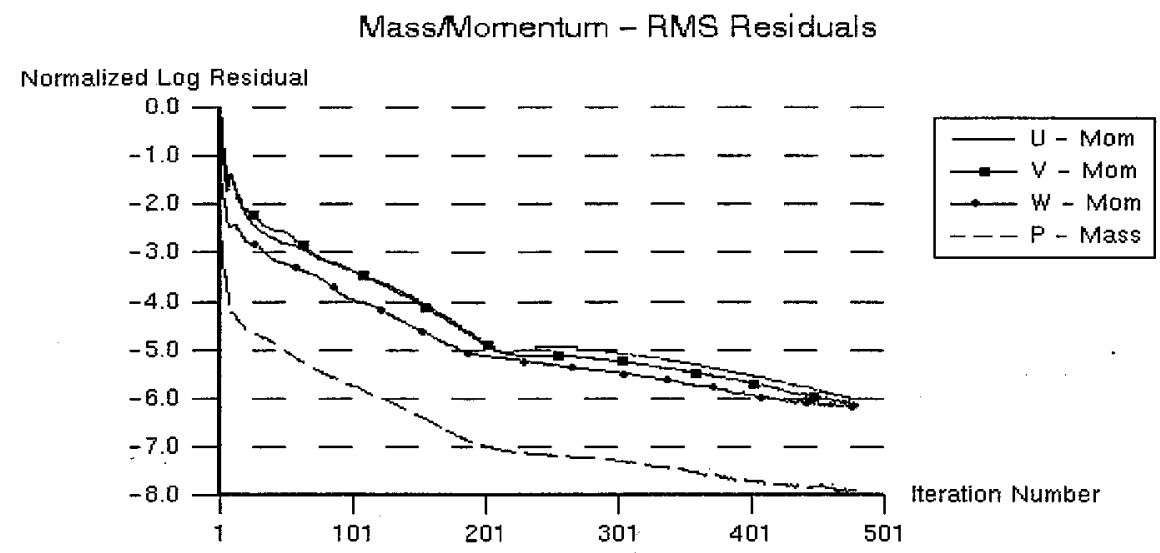

(a)

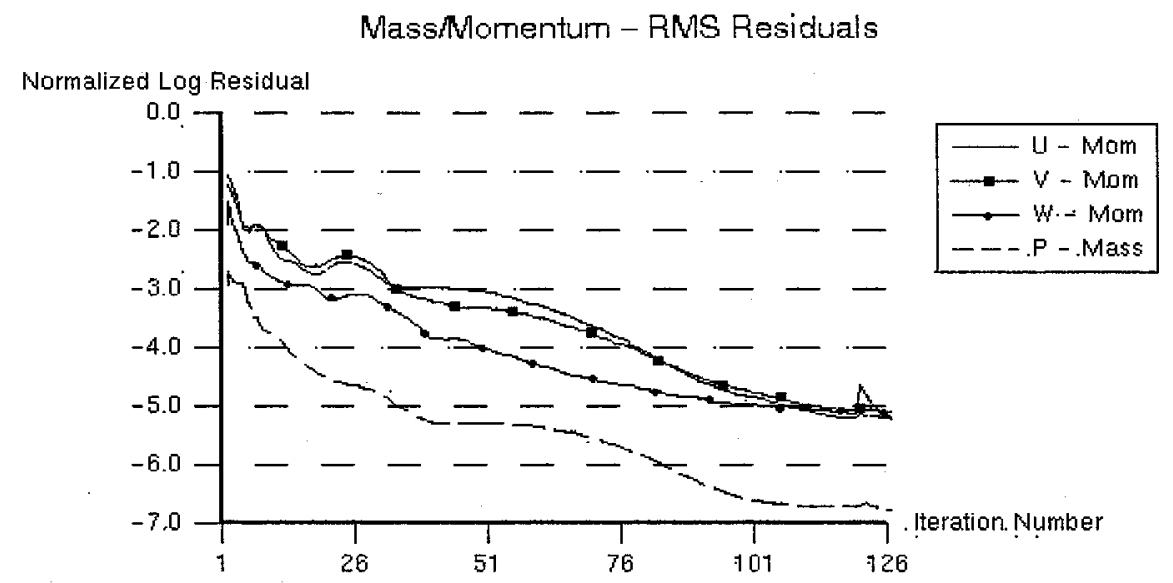

(b)

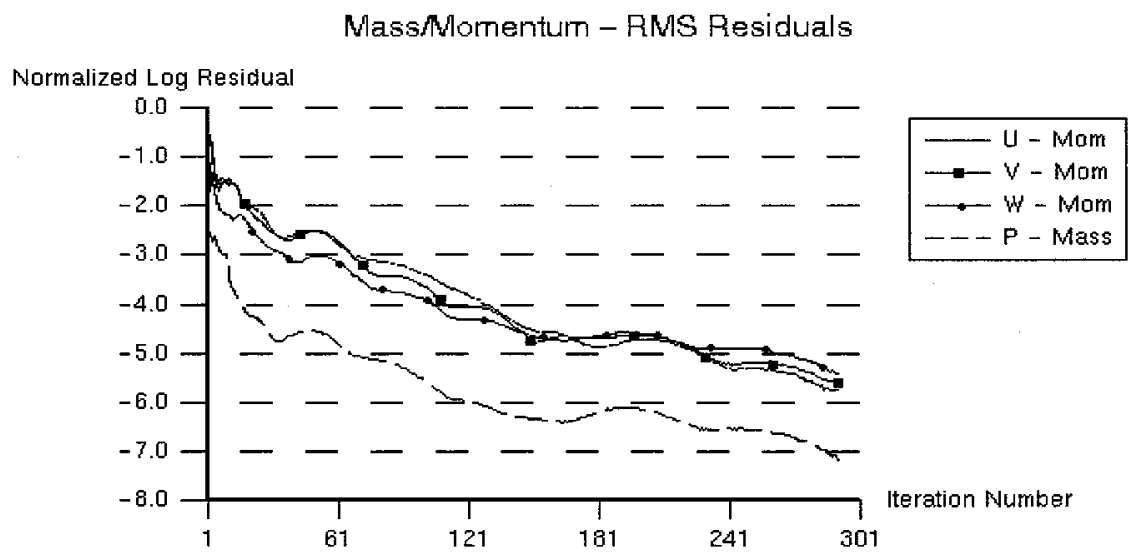

(c)

FIGURE 3

Convergence history for pumps at the design point. (a) Pump M1. (b) Pump M2. (c) Pump M3. 


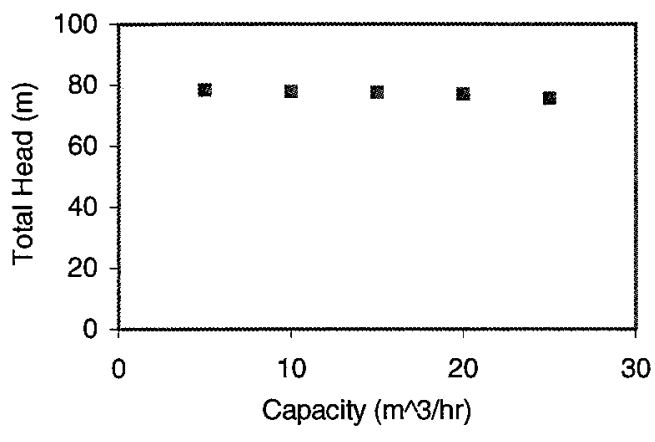

(a)

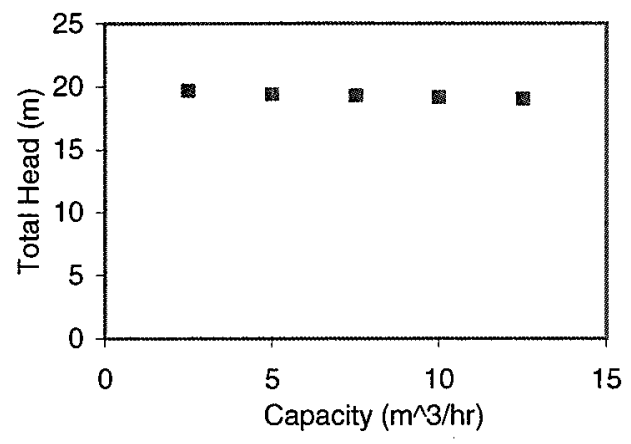

(b)

FIGURE 4

Predicted head-flow curve for pump M1. (a) $\mathrm{n}=2900 \mathrm{rpm}$. (b) $\mathrm{n}=1450 \mathrm{rpm}$.

\section{Boundary Conditions}

The boundary conditions were specified as follows:

- Inlet boundary: A constant mass-flow rate was specified at the inlet of the calculation domain for each computation. Various mass-flow rates were specified so as to study design and off-design pump conditions.

- Solid walls: For the surfaces of the blade, hub, and casing, relative velocity components were set as zero. Also, wall function was applied.

- Outlet boundary: In the outlet of the calculation domain, the gradients of the velocity components were assumed to be zero.

\section{RESULTS AND DISCUSSIONS}

Two rotational speeds-2900 rpm and $1450 \mathrm{rpm}$ - were used in the computations for both the straight-blade and the twistedblade cases. At each rotational speed, several different flow rates were specified at the inlet boundary so as to study design and offdesign flow patterns. Figure 3 shows the convergence histories of pump M1, M2, and M3 at the design point $(\mathrm{n}=2900 \mathrm{rpm})$. The convergence criteria for each run were set to be $1.0 \mathrm{e}-5$ for rootmean-square (RMS) residuals of mass/momentum equations

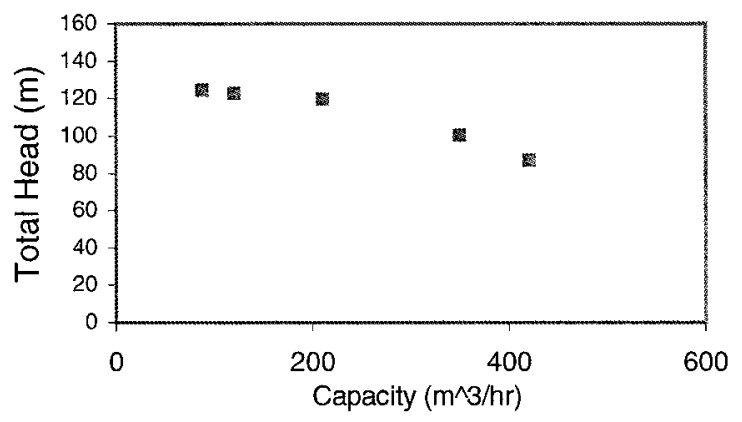

(a) and $1.0 \mathrm{e}-4$ for RMS residuals of $k-\varepsilon$ equations. It was clearly evident that after several hundred time steps in each run, the above criteria could be satisfied, and the convergence was reached gradually.

Figures 4, 5, and 6 show the predicted head-flow curve for pumps M1, M2, and M3 at two different rotational speeds. A good tendency was achieved over the entire flow range for pumps M2 and M3, whereas for pump M1 a deviation was shown for high-inflow volume rate. This suggests that the predicted results of pumps M2 and M3 would be much better than those of pump M1; this may also indicate that the flow was becoming less stable in the last one. The experimental data are not available now; further validation is required by future work.

Figures 7 and 8 show the velocity vectors and pressure distributions on the blade-to-blade plane for pump M1 at the design point and at two rotational speeds, respectively. Similarly, Figures 9 and 10 show velocity and pressure results on the bladeto-blade plane for pump M2, whereas Figures 11 and 12 present the velocity vector and the pressure contour for pump M3. It was found that a severe recirculation occurs in the middle impeller passage in pump M1, whereas in pumps M2 and M3 the flow was much smoother. As for pressure distribution, it can be seen clearly that the pressure increases gradually in a streamwise

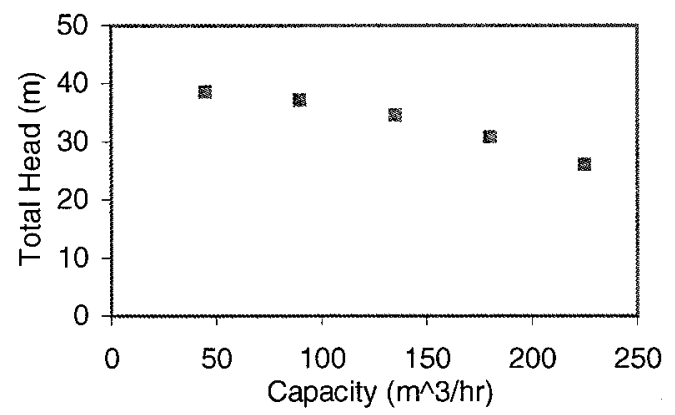

(b)

FIGURE 5

Predicted head-flow curve for pump M2. (a) $n=2900 \mathrm{rpm}$. (b) $\mathrm{n}=1450 \mathrm{rpm}$. 


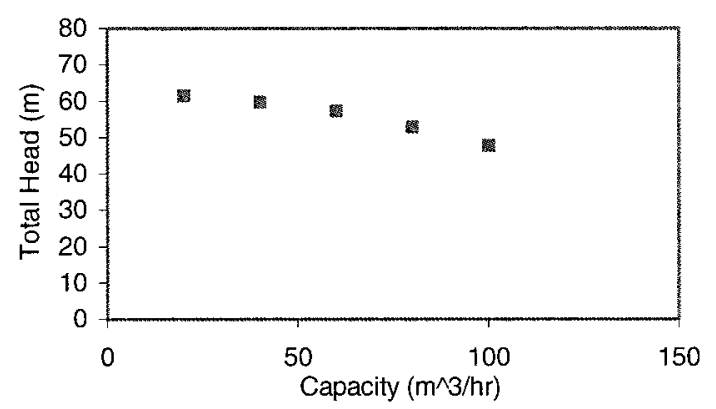

(a)

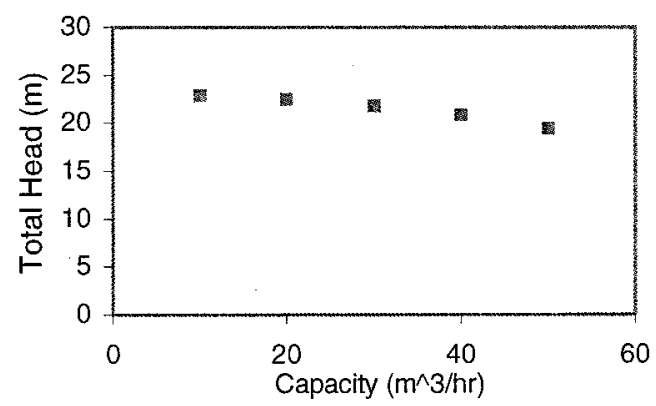

(b)

FIGURE 6

Predicted head-flow curve for pump M3. (a) $n=2900 \mathrm{rpm}$. (b) $n=1450 \mathrm{rpm}$.

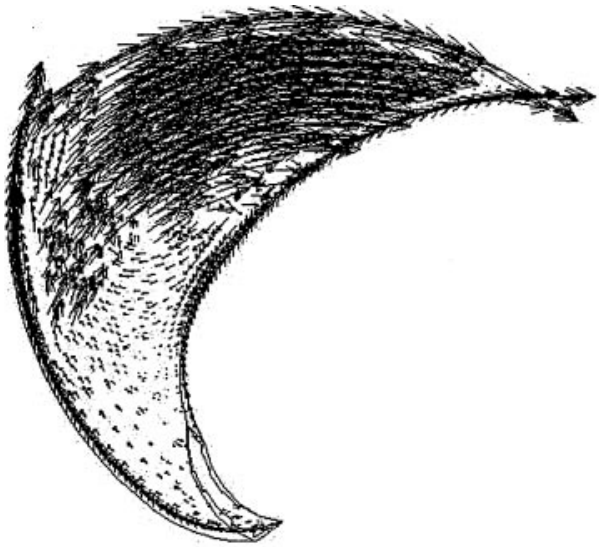

(a)

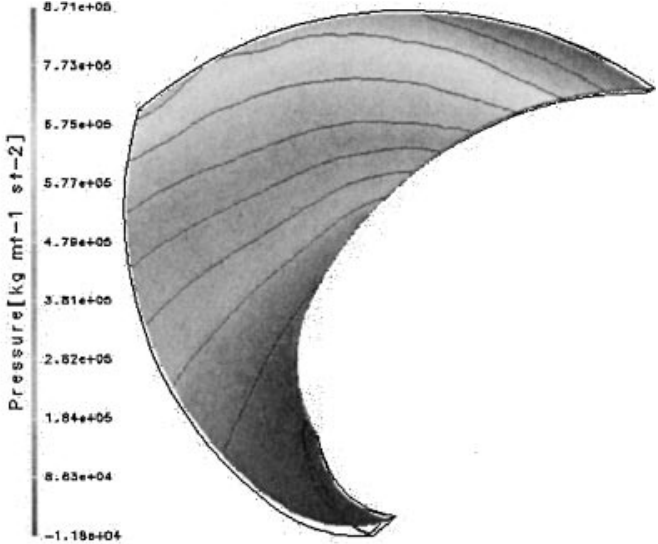

(b)

FIGURE 7

Velocity vectors and pressure distribution on the blade-to-blade plane for pump M1 at the design point ( $\mathrm{n}=2900 \mathrm{rpm}$ ). (a) Velocity vector distribution. (b) Pressure contour.

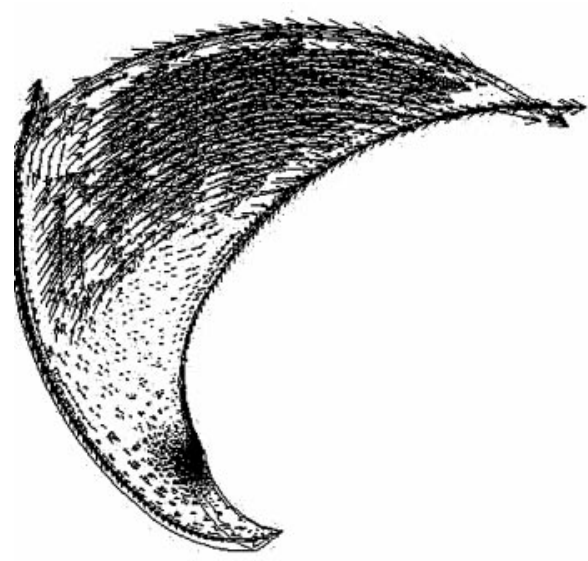

(a)

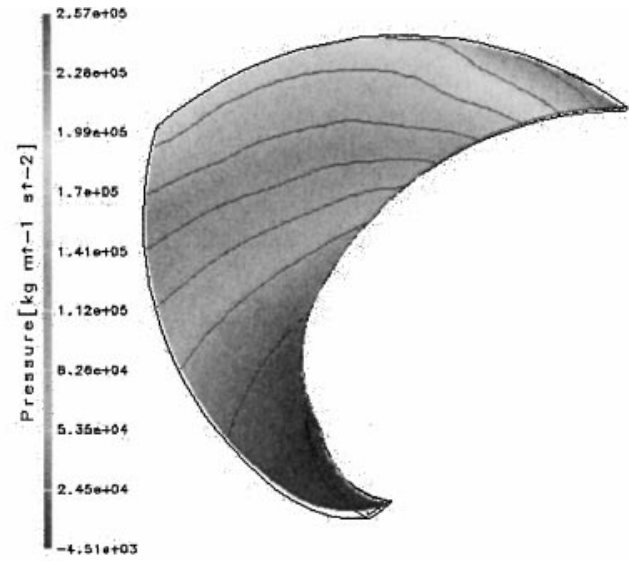

(b)

FIGURE 8

Velocity vectors and pressure distribution on the blade-to-blade plane for pump M1 at the design point ( $\mathrm{n}=1450 \mathrm{rpm}$ ). (a) Velocity vector distribution. (b) Pressure contour. 


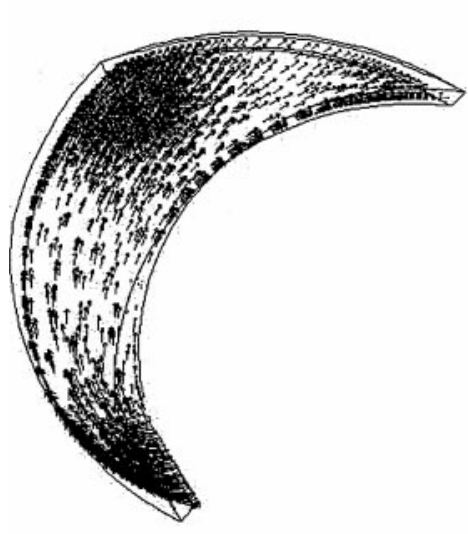

(a)

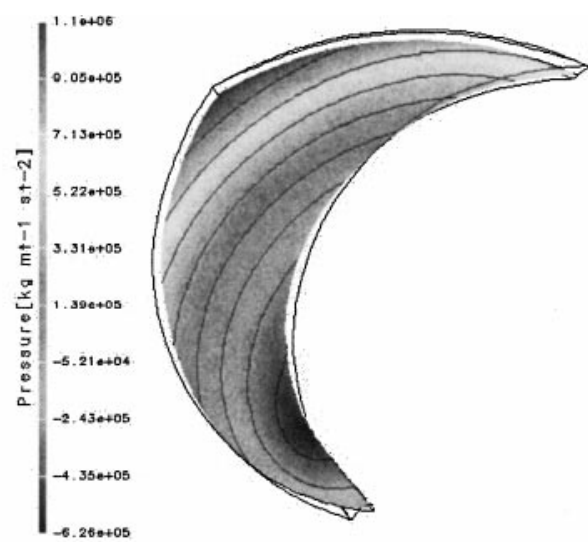

(b)

FIGURE 9

Velocity vectors and pressure distribution on the blade-to-blade plane for pump M2 at the design point ( $\mathrm{n}=2900 \mathrm{rpm}$ ).

(a) Velocity vector distribution. (b) Pressure contour.

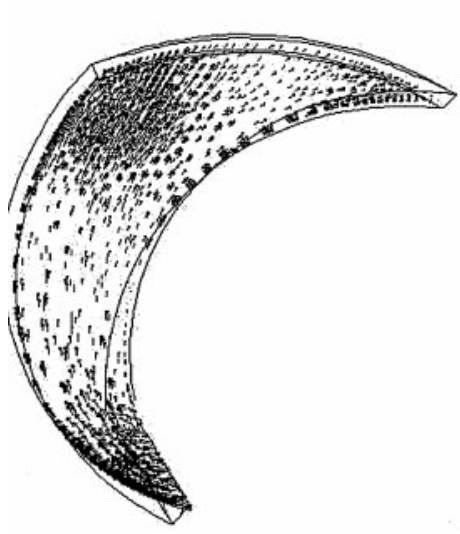

(a)
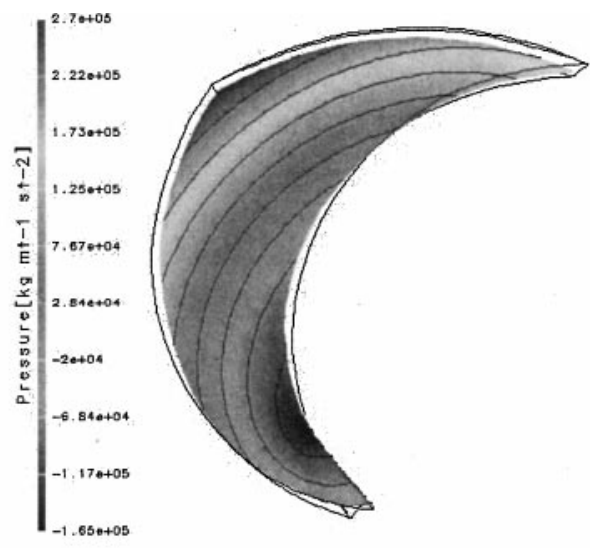

(b)

\section{FIGURE 10}

Velocity vectors and pressure distribution on the blade-to-blade plane for pump M2 at the design point ( $\mathrm{n}=1450 \mathrm{rpm})$. (a) Velocity vector distribution. (b) Pressure contour.

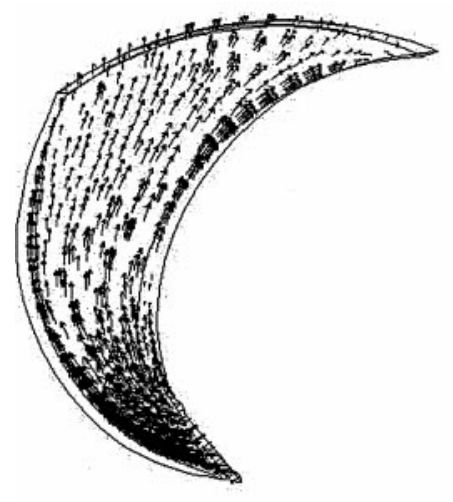

(a)

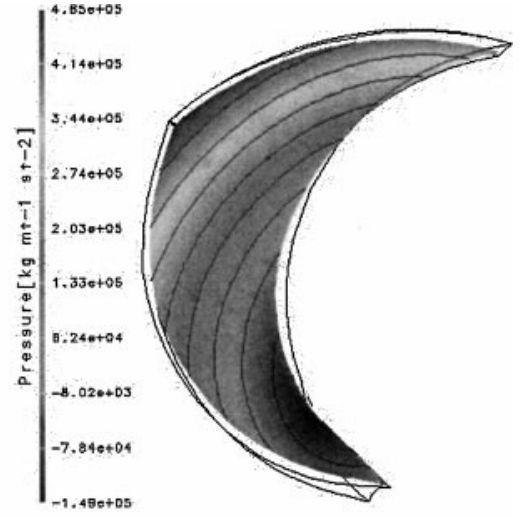

(b)

FIGURE 11

Velocity vectors and pressure distribution on the blade-to-blade plane for pump M3 at the design point ( $\mathrm{n}=2900 \mathrm{rpm}$ ).

(a) Velocity vector distribution. (b) Pressure contour. 


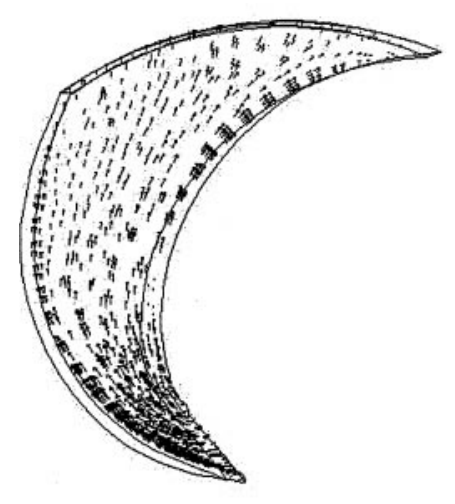

(a)

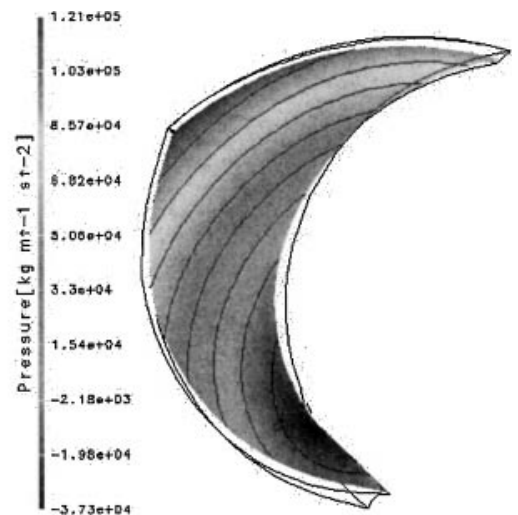

(b)

FIGURE 12

Velocity vectors and pressure distribution on the blade-to-blade plane for pump M3 at the design point ( $\mathrm{n}=1450 \mathrm{rpm})$. (a) Velocity vector distribution. (b) Pressure contour.

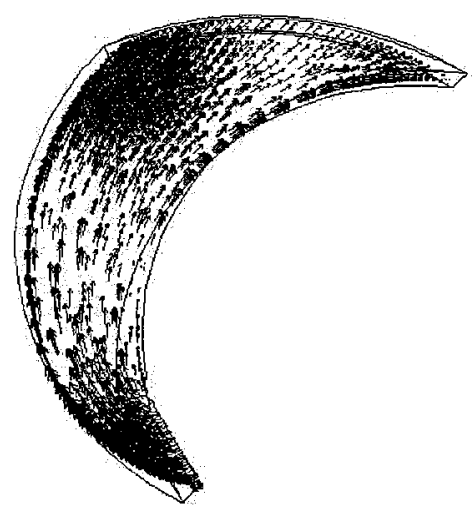

(a)

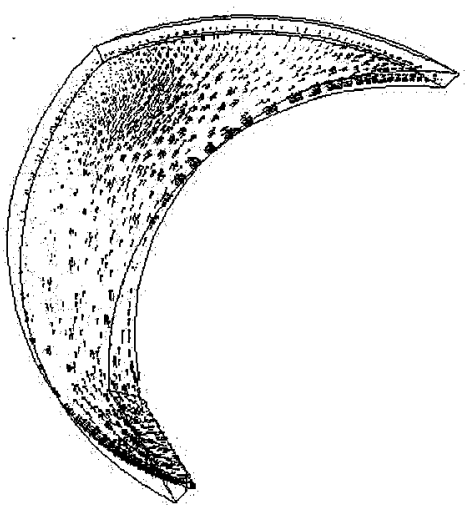

(c)

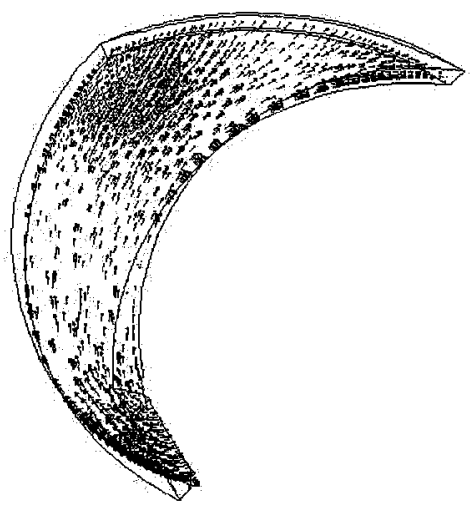

(b)

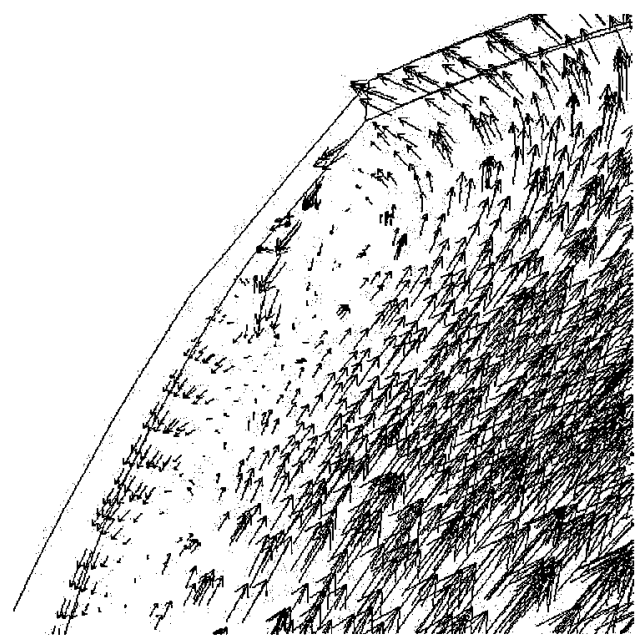

(d)

FIGURE 13

Velocity vector on the blade-to-blade plane for pump M2 at various volume flow rates ( $\mathrm{n}=2900 \mathrm{rpm}$ ). (a) $\mathrm{Q}=420 \mathrm{~m}^{3} / \mathrm{hr}$. (b) $\mathrm{Q}=210 \mathrm{~m}^{3} / \mathrm{hr}$. (c) $\mathrm{Q}=120 \mathrm{~m}^{3} / \mathrm{hr}$. (d) Zone-up view. 
direction, and normally it has higher pressure on the pressure surface than on the suction surface on each plane. But as shown in Figures 7(b) and 8(b), the pressure distribution at the exit near the suction surface was higher than it was in other regions; therefore, reverse flow will occur there as well. All these findings suggest that the efficiency of pump M2 will be better than that of pump M1. Thus, our future work will be focused on improving the design of pump M1.

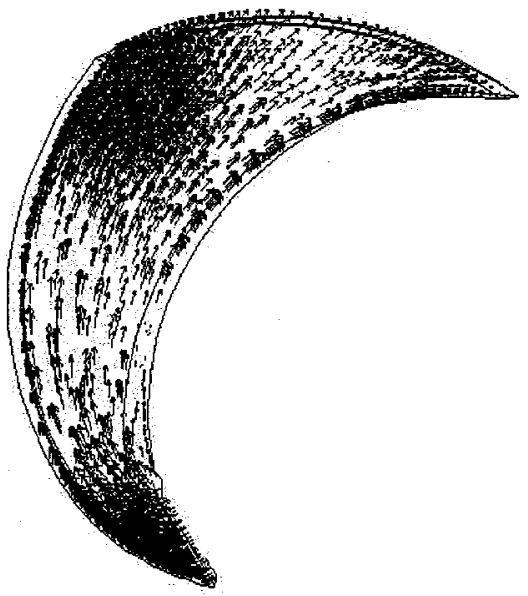

(a)

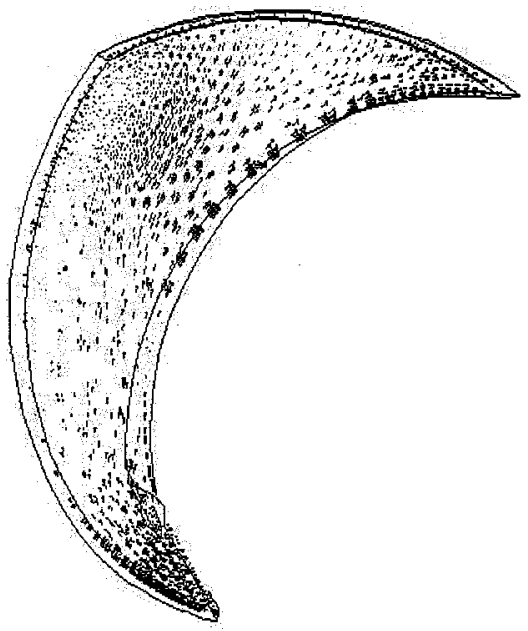

(c)
Various volume flow rates were specified to study off-design conditions for twisted-blade pumps M2 and M3. Figures 13 through 16 show the velocity vectors for these cases at a variety of rotational speeds. It was found that when the inflow rate is within $25 \%$ of the design flow rate, the flow patterns look similar to each other. But if the flow rate drops below a certain value $(35-40 \%)$ of the design flow rate, the flow pattern changes. A strong reverse flow occurs near the pressure surface,

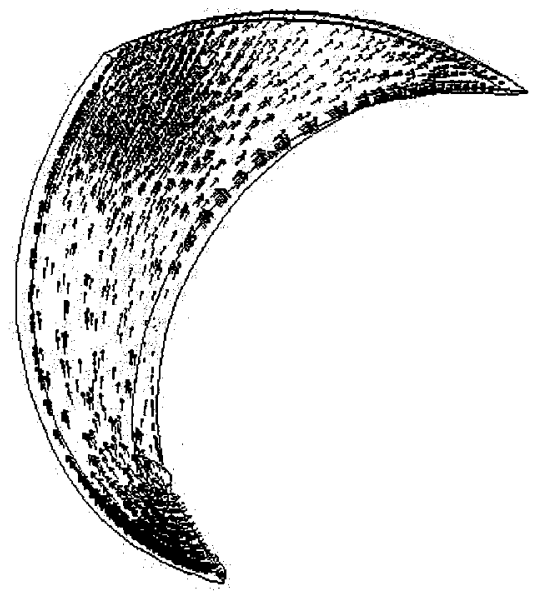

(b)

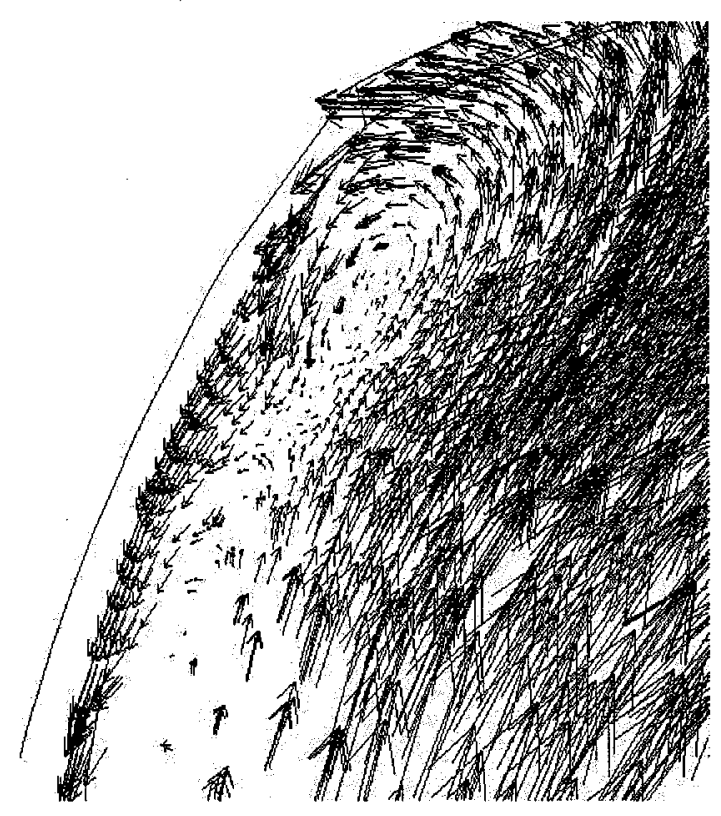

(d)

FIGURE 14

Velocity vector on the blade-to-blade plane for pump M2 at various volume flow rates ( $\mathrm{n}=1450 \mathrm{rpm}$ ). (a) $\mathrm{Q}=225 \mathrm{~m}^{3} / \mathrm{hr}$. (b) $\mathrm{Q}=135 \mathrm{~m}^{3} / \mathrm{hr}$. (c) $\mathrm{Q}=45 \mathrm{~m}^{3} / \mathrm{hr}$. (d) Zone-up view. 


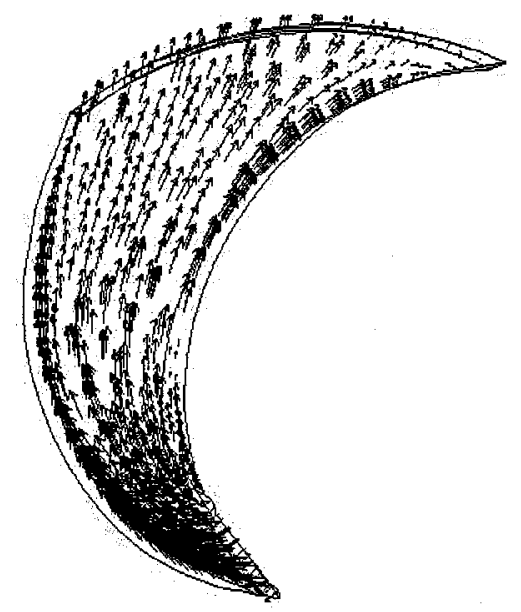

(a)

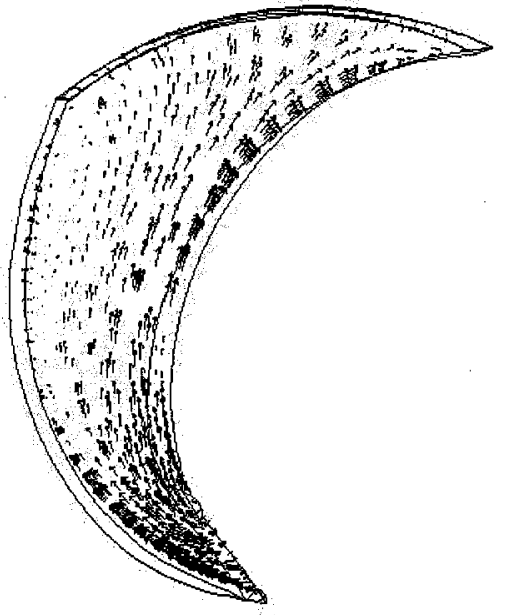

(c)

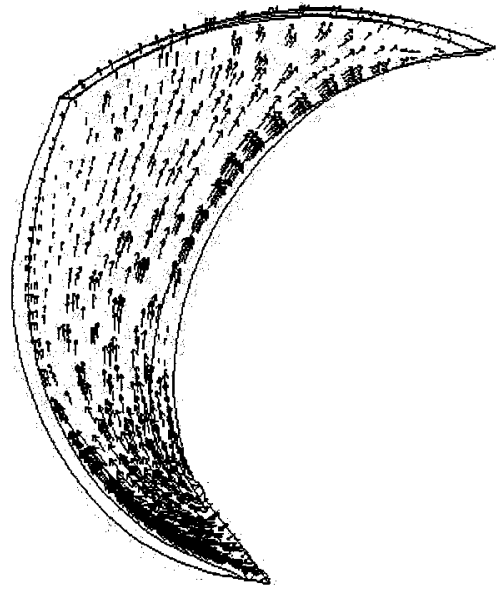

(b)

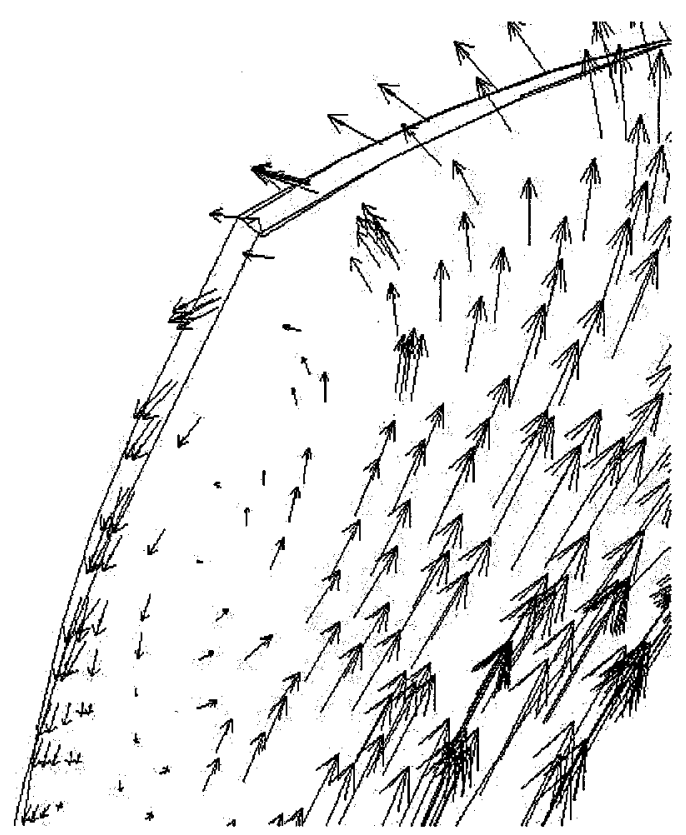

(d)

FIGURE 15

Velocity vector on the blade-to-blade plane for pump M3 at various volume flow rates ( $\mathrm{n}=2900 \mathrm{rpm}$ ). (a) $\mathrm{Q}=100 \mathrm{~m}^{3} / \mathrm{hr}$.

(b) $\mathrm{Q}=60 \mathrm{~m}^{3} / \mathrm{hr}$. (c) $\mathrm{Q}=40 \mathrm{~m}^{3} / \mathrm{hr}$. (d) Zone-up view.

as is shown in Figures 13 through 16c and d. This may occur because when the flow rate through the impeller decreases, the impeller passage correspondingly "narrows" itself so that continuity theory can be satisfied. It can also be seen by referring to Figures 13 through 16 that similar conclusions can be drawn in cases in which a pump operates at different rotational speed.

\section{CONCLUSIONS}

The commercially available three-dimensional NavierStokes code called CFX, which has a standard $k-\varepsilon$ two-equation turbulence model, was chosen to simulate the internal flow of various types of centrifugal pumps-M1, M2, and M3. The predicted results of the head-flow curves are presented over the 


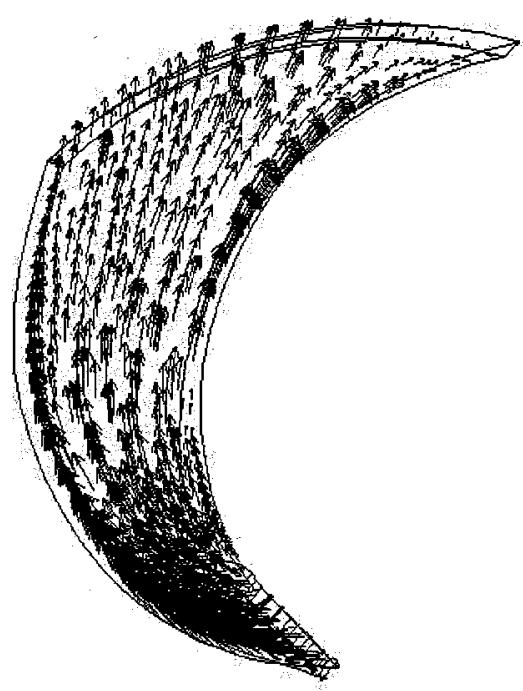

(a)

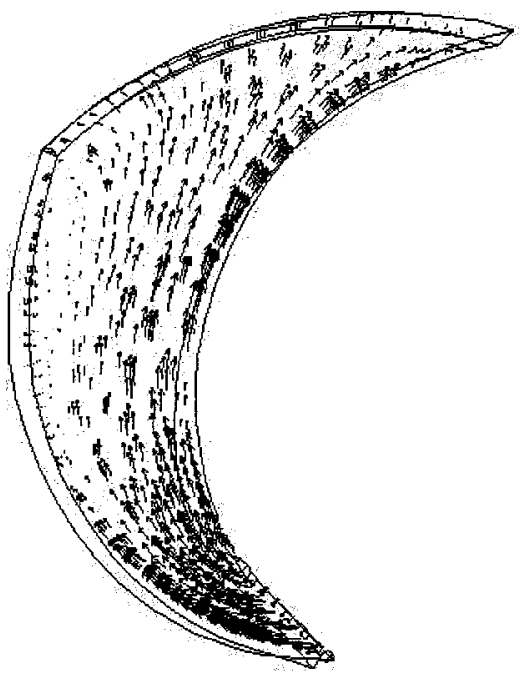

(c)

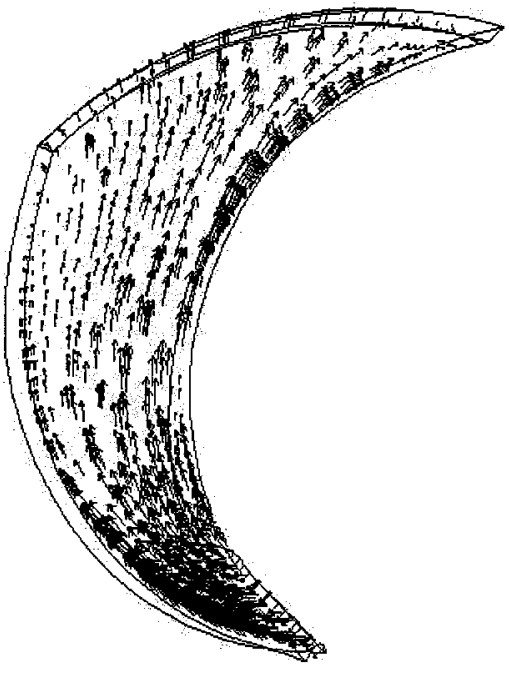

(b)

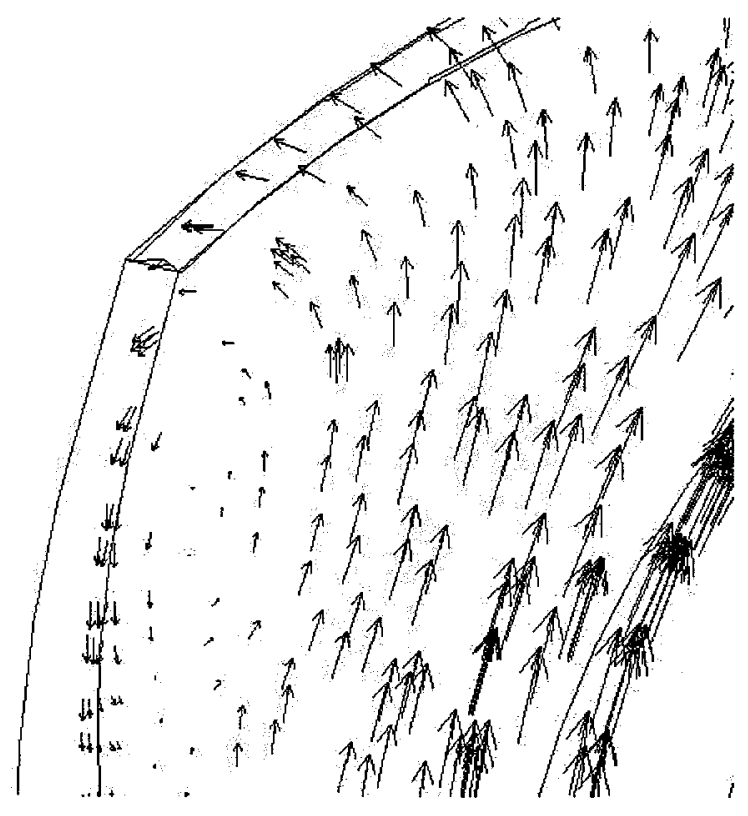

(d)

FIGURE 16

Velocity vector on the blade-to-blade plane for pump M3 at various volume flow rates $\left(\mathrm{n}=1450 \mathrm{rpm}\right.$ ). (a) $\mathrm{Q}=50 \mathrm{~m}^{3} / \mathrm{hr}$. (b) $\mathrm{Q}=30 \mathrm{~m}^{3} / \mathrm{hr}$. (c) $\mathrm{Q}=20 \mathrm{~m}^{3} / \mathrm{hr}$. (d) Zone-up view.

entire flow range. It was found that the predicted results for pumps M2 and M3 were better than those for pump M1, which suggests that the efficiency of pumps M2 and M3 will also be higher than that of pump M1. Thus, future work will be focused on improving the design of pump M1.
This study also shows the flow feature in the off-design condition. It was found that when the flow rate decreased below a certain value of the design flow rate, backflow occurred near the pressure surface of the pump impeller. That might occur because when the flow rate through the impeller decreases, the impeller 
passage correspondingly "narrows" itself so that continuity theory can be satisfied. However, further investigation is necessary to prove that this is so.

\section{NOMENCLATURE}

$l_{t} \quad$ Turbulent length scale

n Rotational speed

$P \quad$ Equivalent pressure

$p_{k} \quad$ Turbulent kinetic energy production term

Q Flow rate

$r \quad$ Location vector

$S \quad$ Source term

$U \quad$ Vector of velocity

$V_{t} \quad$ Turbulent velocity scale

$y^{+}$Dimensionless distance from the wall

$\delta \quad$ The identity matrix

$\varepsilon \quad$ Turbulence dissipation rate

$k \quad$ Turbulence kinetic energy

$\mu \quad$ Molecular viscosity coefficient

$\mu_{\text {eff }}$ Effective viscosity coefficient

$\mu_{\tau} \quad$ Turbulent eddy viscosity coefficient

$\rho \quad$ Density

$\tau_{w} \quad$ Wall shear stress

$\Omega \quad$ Angular velocity

\section{REFERENCES}

Anderson, J., Wood, H. G., Allaire, P. E., and Olsen, D. B. 2000. Numerical analysis of blood flow in the clearance regions of a continuousflow artificial heart pump. Artificial Organs 24:492-500.

CFX5 User Guide. 1996. CFX Computational Fluid Dynamics Software, version 5.3.: AEA Technology, Didcot, UK.

Dawes, W. N. 1986. A numerical method for the analysis of threedimensional viscous compressible flow in a turbine cascade: application to secondary flow development in a cascade with and without dihedral. ASME Paper 86-GT-145. New York: American Society of Mechanical Engineers.

Goto, A. 1992. Study of internal flows in a mixed-flow pump impeller at various tip clearances using three-dimensional viscous flow computations. ASME Journal of Turbomachinery 114:373382.

Kaupert, K. A., Holbein, P., and Staubli, T. 1996. A first analysis of flow field hysteresis in a pump impeller. Journal of Fluids Engineering 118:685-691.

Kolmogorov, A. N. 1941. Local structure of turbulence in incompressible viscous fluid for very large Reynolds number. Doklady Akademiya Nauk SSSR 30:9-13.

Launder, B. E., and Spalding, D. B. 1974. The numerical computation of turbulent flows. Complete Methods of Applied Mechanical Engineering 3:269-289.

Miyazoe, Y., Toshio, S., Ito, K., Konishi, Y., Yamane, T., Nishida, M., Asztalos, B., Masuzawa, T., Tsukiya, T., Endo, S., and Taenaka, Y. 1999. Computational fluid dynamics analysis to establish the design process of a centrifugal blood pump: second report. Artificial Organs 23:762-768.

Ng, E. Y. K., Zhou, W. D., and Chan, W. K. 1998. Non-Newtonian effects on mixed-flow water pump using CFD approach, 735-749. Proceedings of the 19th International Association of Hydraulic Research Symposium, Section on Hydraulic Machinery and Cavitation, Singapore: International Association of Hydraulic Research.

Oh, J. S., and Ro, S. H. 2000. Application of time marching method to incompressible centrifugal pump flow, 219-225. Proceedings of the 2nd International Symposium on Fluid Machinery and Fluid Engineering. Beijing: Tsinghua University Press.

Potts, I., and Newton, T. M. 1998. Use of a commercial CFD package to predict shut-off behavior of a model centrifugal pump: an appraisal. IMechE Seminar Publication: CFD in Fluid Machinery Design. London: Institute of Mechanical Engineers.

Sun, J., and Tsukamoto, H. 2001. Off-design performance prediction for diffuser pumps. Journal of Power and Energy, Proceedings of I. Mech. E, Part A 215:191-201.

Tatebayashi, Y., Tanaka, K., Han, H., and Kobayashi, T. 2000. A 3-D simulation of flow in a screw-type centrifugal pump with tip clearance, 608-615. Proceedings of the 2nd International Symposium on Fluid Machinery and Fluid Engineering. Beijing: Tsinghua University Press.

Zhou, W. D., and Ng, E. Y. K. 1998. 3-D viscous flow simulation of mixed-flow water pump impeller with tip-clearance effects, Proceedings of the 4th International Conference and Exhibition on Pumps and Systems. pp. 189-198. Singapore: HQ Link Pte Ltd. 

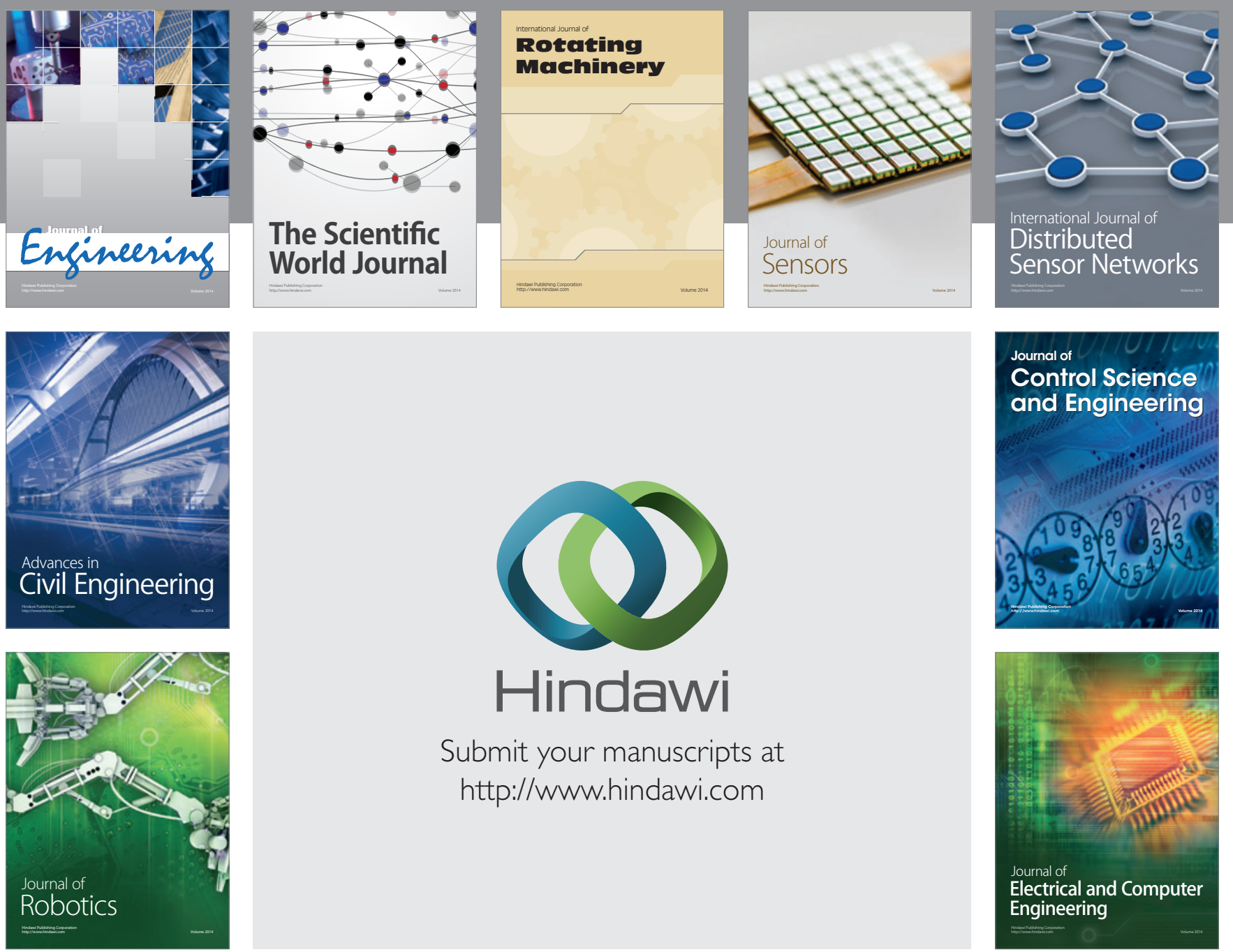

Submit your manuscripts at

http://www.hindawi.com
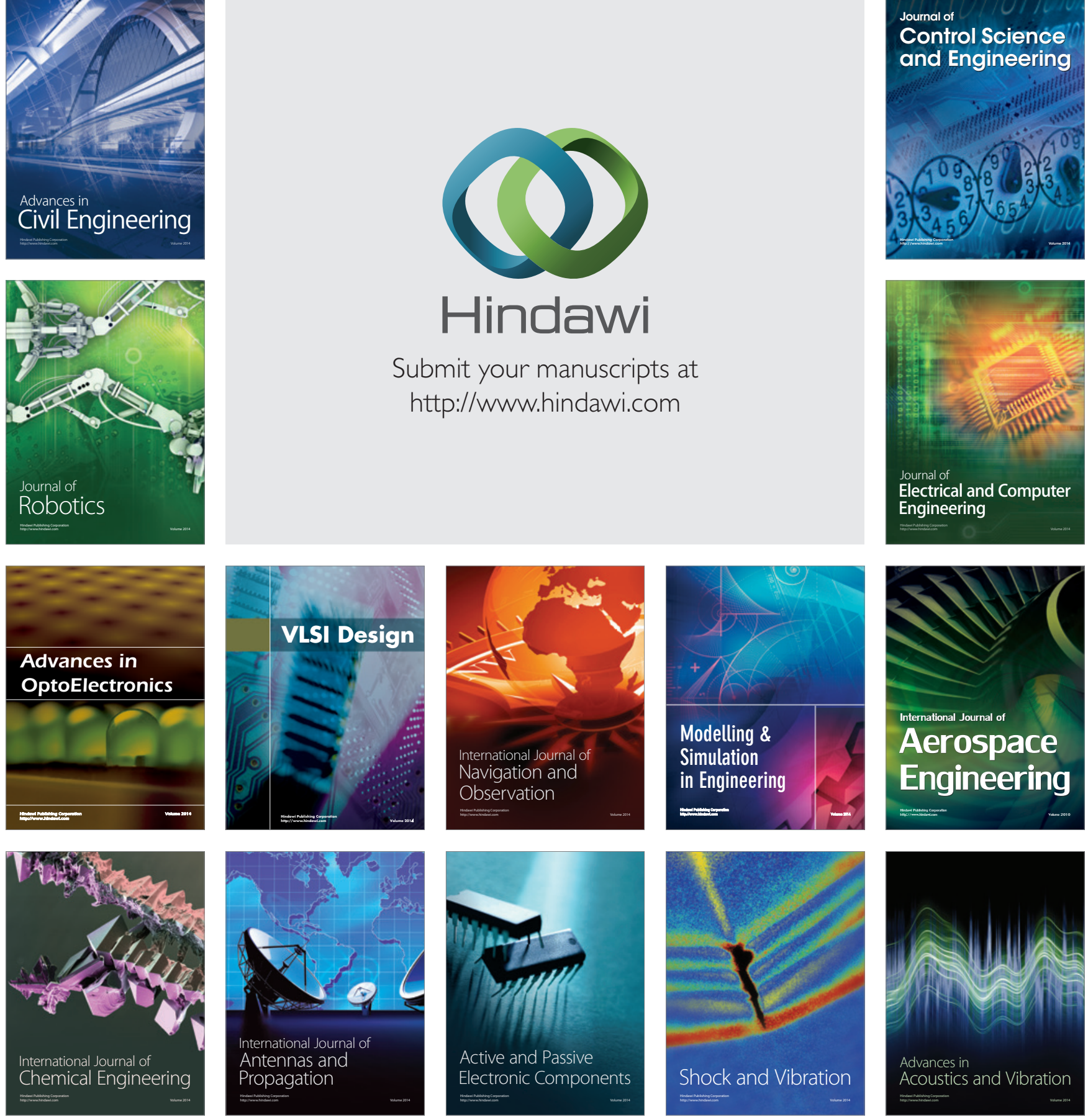